Sportwiss 2015 - 45:57-72

DOI 10.1007/s12662-015-0367-y

Online publiziert: 19. Mai 2015

๑) Springer-Verlag Berlin Heidelberg 2015

\author{
Ralf Brand ${ }^{1}$. Claudia Voelcker-Rehage ${ }^{2}$ \\ ${ }^{1}$ Exzellenzbereich Kognitionswissenschaft, Department für Sport- und Gesundheitswissenschaft, \\ Professur für Sportpsychologie, Universität Potsdam, Potsdam, Deutschland \\ 2 Jacobs Center on Lifelong Learning and Institutional Development, Jacobs University Bremen, \\ Bremen, Deutschland
}

\title{
Ommo Grupe - Nestor der Sportwissenschaft
}

tig ermöglichen uns die herausgebenden Institutionen Bundesinstitut für Sportwissenschaft (BISp), Deutscher Olympischer Sportbund (DOSB) und Deutsche Vereinigung für Sportwissenschaft (dvs), nicht zuletzt durch ihr finanzielles Engagement gemeinsam mit dem Springer Verlag, mittelfristig einen Lebensast. Es gibt Stimmen, die die Sportwissenschaft vor allem Ausdruck der an bundesdeutschen Hochschulen und in deutscher Sprache kommunizierten Sportwissenschaft sein lassen wollen. Andere zweifeln grundsätzlich an der Idee eines einheitlichen Faches Sportwissenschaft und würden auch auf die dazugehörige Zeitschrift verzichten. Und schließlich gibt es Jene, die eine viel konsequentere Öffnung zur internationalen Scientific Community für vernünftig halten, sich für eine Sportwissenschaft einsetzen, die vor allem auch englischsprachig publizierende Kolleginnen und Kollegen anspricht, und diese als neue Beitragende für unsere Zeitschrift gewinnen wollen.

Schon die hier genannten, aber auch eine ganze Reihe weitere, uns vorgetragene Positionen, sind in ihren Konsequenzen kaum miteinander zu verbinden. Interessant finden wir zu bemerken, dass die aktuelle Situation der Sportwissenschaft der Zeitschrift genauso wie die der Fachdisziplin - zumindest in Ausschnitten der 1971 von Ommo Grupe beschriebenen gleicht. Ein solcher Ausschnitt sei im Folgenden kurz beleuchtet.

\section{Das Ganze ist mehr als die Summe seiner Teile}

Eine bemerkenswerte These, im Gründungseditorial ist unseres Erachtens die, dass das Auffächern eines disziplinären Fächerspektrums und die Zusammenführung entsprechender Fachbeiträge im Rahmen der damals neu gegründeten Zeitschrift, die Entwicklung von Methoden und Fragestellungen für wissenschaftlich fundierte interdisziplinär sportwissenschaftliche Betrachtungen fördern möge, und dass sich daraus schließlich eine Theorie des Sports formieren könne, die Voraussetzung für ein (dann) ernstzunehmendes akademisches Fach Sportwissenschaft sei. Ommo Grupe unterstreicht dabei, dass sich Sportwissenschaft davor hüten sollte zufrieden zu sein mit spezialdisziplinären Betrachtungen, die durch das gemeinsame Beiwort Sport Gemeinsamkeit nur vorspiegeln und die ergo darüber hinwegtäuschen, dass eigentlich „nur“ Psychologie, Pädagogik, Medizin oder Biomechanik (u. w.) gemacht wird. Er intendierte „seine“ Zeitschrift 1971 als Instrument, welches dem Zweck dienen sollte, Sportwissenschaft als integrative, vor allem aber eigenständige Fachdisziplin zu etablieren. Den Titel der neuen Zeitschrift, Sportwissenschaft, nicht Sportwissenschaften, bezeichnete er als „Programm und einzulösender Anspruch zugleich“.

Wo steht die Sportwissenschaft heute?

Das Fach Sportwissenschaft hat sich an deutschen Universitäten und Hochschulen etabliert. In den Denominationen der dortigen Professuren oder am SektionenSpektrum der dvs spiegelt sich die Vielfältigkeit der unter dem Dach Sportwissenschaft mittlerweile zusammengeführten Fachdisziplinen wider. Dieselbe Vielfalt bildet sich auch durch die in der Sportwissenschaft publizierten Beiträge ab.
Substanz der Zeitschrift beitragen könnten, in der Zahl mangelhaft. Gleichzei- 


\section{Einleitung in die „Sportwissenschaft"}

(oder: Über die Schwierigkeit, eine neue Publikation zu planen)

Nach jahrelangen Verhandlungen und ebenso langer organisatorischer, aber viel zu kurzer inhaltlicher Planung erscheint nun, fast schon unerwartet, die erste Nummer der "Sportwissenschaft" - das möglicherweise zu früh geborene Kind einer wissenschaftlichen Disziplin (das Datum der Zeugung ist nicht bekannt, um die Vaterschaft streitet man sich noch in DSB und ADL), von der man noch nicht weiß, ob sie die nun auf sie entfallende Vater- und Mutterrolle angemessen ausfüllen kann. Sei's drum - die erste Nummer liegt nun vor, nicht so gut, wie die Planer erhofften, nicht so schlecht, wie Kritiker voraussagten, gewiß aber ein Spiegelbild dessen, was Sportwissenschaft im Moment zu bieten vermag. Daß dies mehr, breiter fundiert, noch gründlicher, empirisch gesicherter werde, in kritischer Absicht geschehe und zur Aufklärung über individuelle und soziale Zusammenhänge des Sports und zu seiner ständigen Verbesserung beitrage, ist ein Wunsch, der sich mit dem erstmaligen Erscheinen der "Sportwissenschaft" verbindet.

Freilich ist es mit solchem Wunsch nicht getan. Nicht nur Eingeweihte oder dem Sport gegenüber Skeptische wissen, daß er sich traditionell nicht gerade als theoriefreundlich gezeigt hat; selbst zu dem, was man überhaupt als eine Theorie bezeichnen konnte, hatte er - seine Institutionen, seine Verbände, seine Bürokratie - in aller Regel ein eher gebrochenes Verhältnis. Wenn Theorie schon nicht zur Bestätigung für Bestehendes oder zur Rechtfertigung von ohnehin Gewolltem dienen konnte, vielmehr verunsicherte und Traditionelles, Liebgewordenes, Gewohntes aufs Spiel setzte, was sollte sie dann?

Nicht allein den Apologeten des Sports, auch seinen Gegnern mußte ein solches Verhältnis zur Theorie sympathisch sein, lieferte es doch Ansatzpunkte für Kritik in einer Fülle, daß sie es sich leisten konnten, ihre (Vor-) Urteile der empirischen Uberprüfung und ihre Hypothesen dem kritischen Zugriff zu entziehen; vor dem Anspruch kühner, kultur- und gesellschaftskritisch motivierter Verwerfung des Sports mußte - angesichts der offenkundigen Schwächen seiner theoretischen Begründung - jedes Gegenargument zum kleinlichen Widerspruch degenerieren; selbst der Hinweis auf das abweichende empirische Detail fügte sich dem großen Konzept als hübsche Variante. Die undifferenzierte Verallgemeinerung von Einzel- 


\section{Ommo Grupe}

aussagen, die Ignorierung der unterschiedlichen Teilbereiche und Funktionen des Sports und ihre ständige Verwechslung, die Hypostasierung von Details ist insofern nicht nur Kennzeichen derer, die vorgeben, ihm zu helfen, sondern auch mancher seiner Kritiker.

Solange Zweifel an der Notwendigkeit einer engen Beziehung zwischen Theorie und Praxis bestanden, der - wenn man will - dialektische Zusammenhang zwischen Wirklichkeit und Analyse dieser Wirklichkeit gering veranschlagt werden konnte, mochte man sich mit einem saloppen Theorie- und Wissenschaftsverständnis abfinden; wenn jedoch enge und notwendige Verflechtungen akzeptiert werden, so entstehen andere Zweifel, ob man z. B. gut beraten war, die Sportwissenschaft so lange dem zufälligen und privaten Interesse von einigen Medizinern und Pädagogen zu überlassen, anstatt die für die Entwicklung einer Wissenschaft erforderlichen methodologischen Vorüberlegungen anzustellen und institutionellen Voraussetzungen zu schaffen. So nämlich konnte sich die Meinung verfestigen, Sport bedürfe eigentlich keiner Theorie; er sei eine eher praktische Disziplin und als solche schon Theorie genug. Theorie sei allenfalls als jene Art vorwissenschaftlicher Erfahrung legitim, die helfe, der Praxis gestellte Ziele auf ökonomischstem Wege zu verwirklichen, ohne sie selbst aber grundsätzlich in Frage zu stellen. Die Folgen einer solchen Auffassung zeigen sich in dem ohne Zweifel niedrigen Standard sportwissenschaftlicher Forschung in der Bundesrepublik (was gewiß auch andere Gründe hat, so z. B. die mangelhafte Nachwuchspflege, die zu späte Etablierung sportwissenschaftlicher Forschung an den westdeutschen Hochschulen, auch die selbstgenügsame Isolierung der Vertreter der Leibeserziehung) und dem häufig affirmativen Charakter ihrer Aussagen, die sich nicht selten als jene Art von Glaubenssätzen erweisen, die der gängigen Praxis von vornherein, nicht aber auf der Grundlage kritischer Überprüfung Absolution erteilen - Wissenschaft sozusagen als Alibi.

Jedoch ist längst deutlich geworden - und sowohl der Deutsche Sportbund als auch eine Reihe von Hochschulen haben daraus Konsequenzen gezogen - , daß ein Bereich von solcher Dynamik und solchem öffentlichen Gewicht, wie er sich im Sport (und der Freizeit) präsentiert, auf die Dauer nicht mehr auf der Grundlage ungeprüfter Erfahrungen und ungenauer Allgemeinvorstellungen erfaßt, geplant, verändert werden kann. An dem Punkt, an dem erfahrungsgeleitetes Handeln in langfristige Planung und zum Entwurf weitreichender Perspektiven übergehen muß, bedarf es rational-kritischer Analysen und empirischer Forschungen. In einer Gesellschaft, deren Zukunft in einem so erheblichen Maße von den Lei- 
stungen ihrer Wissenschaftler abhängig ist wie die gegenwärtige, bedarf auch die Entwicklung des Sports in seinen verschiedenen Teilbereichen der sie begleitenden und fundierenden Forschung, um sie vor Fehlläufen, Mißverständnissen, Ideologien zu schützen und konkrete Planung im Kontext von sport-, bildungs- und auch gesellschaftspolitischen Entscheidungen zu ermöglichen. Zur Wirklichkeit des Sports gehört heute die differenzierte Analyse dieser Wirklichkeit, zu seiner Praxis die kritische Reflexion dieser Praxis, damit sie akzeptiert, verbessert und gegebenenfalls auch verworfen werden kann. Dem guten, wenn nicht besten Sinn von Wissenschaft ist zuzurechnen, neben der unbefangenen, undogmatischen Erkenntnis von Wirklichkeit Impulse zu ihrer Verbesserung auszulösen.

\section{Die Vielschichtigkeit des Sports und die Notwendigkeit, ibr zu entsprechen}

Dabei ist kein $Z$ weifel, daß der Sport im sozialen Leben der Gegenwart zu jenen auffälligen, wenngleich in ihren Wirkungen und Strukturen noch unausgemessenen Erscheinungen gehört, über die globale Aussagen noch gar nicht, Teilaussagen nur in begrenztem Maße möglich sind. Freilich ist er auf den ersten Blick unkompliziert, unproblematisch, durchsichtig in seinen Strukturen und verständlich in seinen zutage liegenden Zusammenhängen; seine gelegentlich archaische Intensität und ungebrochene Anziehungskraft haben ihren Grund in solcher kommunikationsfreundlichen Transparenz. Und doch kann dies nicht darüber hinwegtäuschen, daß er im Zusammenhang individuellen und sozialen Lebens und in seinen gesellschaftlichen Verflechtungen noch weitgehend unerforscht ist. So mag es zu erklären sein, daß er nicht frei von Mißdeutung, Fehlinterpretation und ideologischer Verbrämung geblieben ist. Das systematische Interesse gediegener Forschung hat ihn kaum erreicht. Gewiß vermag die Medizin einiges (und teilweise eine Menge) über seine gesundheitlichen, d. h. prophylaktischen, rehabilitativen oder therapeutischen Wirkungen auszusagen; was aber z. B. nur die vielfältigen Beziehungen zwischen sozialer Wirklichkeit und Sport oder was seine Erziehungsmöglichkeiten angeht, sind wir auf empirisch wenig gesicherte Annahmen angewiesen. Die Dürftigkeit der wissenschaftlichen Ergebnisse steht in einem merkwürdigen Widerspruch zu der sichtbaren öffentlichen Bedeutung und Inanspruchnahme des Sports.

So mischen sich apologetische Äußerungen mit undifferenzierten Bewertungen, unerfüllbare Erwartungen und unbegründete Hoffnungen mit kritischen Ansätzen jedweden Niveaus, unterschiedlicher Couleur und auf einer Bandbreite, die von sport- und gesellschaftsimmanenter Kritik bis hin zur totalen Kritik am Sport mitsamt dem System reicht, dem er zugehört: Kritik an Auswüchsen des Sports, an seiner Kommerzialisierung, seiner politischen und ökonomischen Inanspruch- 


\section{Ommo Grupe}

nahme, an den von ihm ausgelösten oder begünstigten Massenphänomenen, die oft vorschnell als Symptome der Verkörperlichung und des geistigen Verfalls diagnostiziert werden, schließlich Kritik am Sport überhaupt als einem probaten Mittel der jeweils Herrschenden, ihre wahren Absichten zu verbergen und die Sporttreibenden auf kleinbürgerliche Verhaltensmuster der Bravheit und des Gehorsams zu prägen. Vorurteile, spätbürgerliche Ressentiments, bildungsbürgerliche Traditionen wechseln in lockerer Folge mit pauschaler Verteidigung und romantisierender Selbstdeutung des Sports als eines Stïcks (noch) konfliktloser Welt. Von einer geschlossenen Beurteilung des Sports und seiner verschiedenen T'eilbereiche sowie einer in sich überzeugenden Theorie ist dies alles ebenso weit entfernt wie von anspruchsvoller Kritik - es sei denn, man akzeptiert Aperçus schon als solche. Es fehlt häufig an Augenmaß, an kritischer Distanz, oft aber auch an der Bereitschaft, sich ernsthaft einzulassen mit einem Phänomen, dessen Massencharakter und gleichsam proletarische Herkunft - sofern es sich nicht gerade um Herrensportarten (und ums IOC) handelt - immer noch eine Abdichtung gegenüber Wissenschaft bedeuten. So gesehen ist eine Differenzierung des Sports nach seinen Teilgebieten, deren - soweit möglich - präzise Abgrenzung und ihre inhaltliche Unterscheidung nach den Funktionen, die sie innerhalb bestimmter sozialer Gruppen oder Systeme haben - anstatt global vom Wesen des Sports zu reden und ihm Ziele einfach zu unterstellen -, für die "Sportwissenschaft ${ }^{\text {“ }}$ eine wichtige Aufgabe. Allerdings macht sie erhebliche Schwierigkeiten.

\section{Systematisierungsprobleme}

Der Versuch einer Systematisierung der Sportwissenschaft, wenn sie schon nicht als einheitliche Disziplin akzeptiert werden kann, die sinnvollerweise auch der "Sportwissenschaft" zugrunde gelegt würde, hat seine Probleme sowohl in der Komplexität des Forschungsgegenstands als auch in der Unterschiedlichkeit der Methoden und Betrachtungsweisen, die sich als auf diesen Gegenstand anwendbar erweisen. Genau genommen stellen diese jedoch nicht das methodologische Hauptproblem dar. Auch für die Sportwissenschaft zeigen sie sich eher als traditionelle Probleme, für deren Lösung es in anderen wissenschaftlichen Disziplinen bereits eine Reihe von Vorbildern gibt. Das Hauptproblem der Sportwissenschaft stellt sich - soweit dies abzusehen ist - vor allem darin, von ihrer Systematik, ihrer Fragestellung und ihren methodischen Ansätzen aus sicherzustellen, daß ihr Anspruch auf interdisziplinäre Forschung eingelöst wird. Sicherlich gibt es nach wie vor eine Reihe von Problemen des Sports, die auch von den Fragestellungen und Verfahrensweisen anderer wissenschaftlicher Disziplinen abgedeckt werden kön- 
Einleitung in die „Sportwissenschaft ${ }^{\star}$

nen; jedoch bleibt dies in der Regel auf die von ihrem Ansatz her vorgezeichneten Problemausschnitte beschränkt, ohne den Sport als ein Problem verschiedener wissenschaftlicher Disziplinen zu erreichen. Ginge die Sportwissenschaft - wie das noch weithin geschieht - von der konventionellen Aufteilung nach wissenschaftlichen Disziplinen aus, so fiele sie prompt auf eine Aufteilung nach pädagogischen, psychologischen, soziologischen, medizinischen und anderen Fragestellungen zurück, wobei diese durch das Beiwort „Sport-“ zwar eine Gemeinsamkeit des Forschungsgebiets vorspiegeln, tatsächlich jedoch nur über das nicht realisierte integrative Moment hinwegtäuschen. Das gleiche gilt für die zweite - gleichfalls traditionelle - Aufteilungsmöglichkeit, nämlich die Systematisierung anhand der organisatorischen Schwerpunktbereiche des Sports, in denen er sich vorwiegend darstellt. Als solche Bereiche werden z. B. immer wieder angeboten:

Leibeserziehung/Schulsport/Jugendsport als die nach im weitesten Sinn pädagogischen Kriterien ausgewählten, akzentuierten und eingesetzten Formen der Leibesübungen und des Sports (Vorschulerziehung, Primarstufe sowie Sekundarstufe I und II; Hochschule; außerschulische Erziehung; Jugendverbände; sozial- bzw. sonder- und heilpädagogische Maßnahmen; Heimerziehung; Resozialisation);

Breitensport/Freizeitsport/Sport für alle: Formen des Sports und der Leibesübungen also, die Spiel und Bewegung von Kindern, Jugendlichen, Erwachsenen und alternden Menschen umfassen (Verein; kommunale Einrichtungen, Betriebssportgruppen, Volkshochschulen; unorganisierte Sportaktivitäten; sportlich akzentuierte Wochenend- und Urlaubsveranstaltungen);

Leistungs- und Wettkampfsport als Sport von Individuen und Gruppen, die sich regelmäßigem, geplantem Training und Wettkämpfen unterziehen;

Höchstleistungssport/Spitzensport als ein strukturell abhebbarer Sportbereich, der sich in seinen Zielen und Verwertungszusammenhängen politischer und ökonomischer Art als deutlich unterscheidbar vom Breitensport erweist und in seinem Bestand in sehr viel höherem Maße auf Zuschauer und öffentliches Interesse angewiesen ist als Breiten- und Wettkampfsport. Der Ubergang vom Höchstleistungssport zum Berufssport, Professionalismus, liegt nahe, sobald ein Markt sich bildet und das Publikumsinteresse steigt, ein Teil, wenn man will, moderner Unterhaltungszweige.

Wie immer man diese Aufteilung auch vornimmt und wie weit man die Differenzierung der Teilbereiche des Sports auch treibt, im Grund bleibt eine solche Systematisierung in einem konventionellen Verständnis von Sport und dem traditio- 


\section{Ommo Grupe}

nellen Begriff von Wissenschaft stecken. Von einem integrativen Ansatz aus wäre es hingegen konsequent, solche Kriterien zu ermitteln, die quer durch wissenschaftliche Fragestellungen und organisationsbedingte Aufteilungen hindurch eine eher mehrdimensionale Klassifikation ermöglichen. Mehrdimensionalität heißt in diesem Zusammenhang, daß im Bereich des Sports die durchgängigen (horizontalen) Fragestellungen akzentuiert werden, jedoch nicht so, daß sich organisatorische Unterschiede zwischen Teilbereichen des Sports verflüchtigen und Methoden und Verfahrensweisen bestimmter Disziplinen undifferenzierter Verwendung anheimgegeben werden. Als solche Fragen bieten sich z. B. das Problem der Leistung, des Konsums, des Zuschauens, der Beanspruchung und Belastung, der politischen und wirtschaftlichen Verwertung des Sports, der Gruppendynamik, der Motivation an. Mit ihrer Hilfe mag sich ein mehrgliedriges Raster entwickeln lassen, das gröBere Präzision in der Bestimmung der Inhalte, Strukturen, Funktionen und Möglichkeiten des Sports (und seiner Teilgebiete) erlaubt. Größere Genauigkeit kann aber auch wohl nur der einzige einsehbare Sinn von Klassifikationen und Systematisierungsversuchen sein.

Die "Sportwissenschaft" wird versuchen, ihre eigenen inhaltlichen Planungen an einem so differenzierten und noch präziser zu differenzierenden Gegenstandsbereich „Sport" auszurichten, ihre Fragestellungen theoretischer und empirischer Art auf diese abzustellen, zugleich aber - und möglichst vor allem - immer wieder diejenigen Fragen zu reflektieren, die die Teilbereiche horizontal durchschneiden und insofern diejenigen Kriterien freigeben, die für ihre Forschungsansätze fundierenden Charakter besitzen; dies werden vor allem anthropologisch-philosophische, sozial- und kulturanthropologische sowie sozial- und verhaltenswissenschaftliche und allgemein-medizinische Themen sein. Dabei zeigt sich auch, daß diese Problembereiche wiederum in sich so vielschichtig sind, daß sie nicht einem Teilgebiet der Sportwissenschaft zugeordnet werden können (jedenfalls wäre eine Zuordnung durchweg nicht eindeutig vorzunehmen), sondern in der Regel differenzierter und zugleich integrativer Verfahren bedürfen.

Wiederum setzt solche Differenzierung eine Differenzierung nach den Interessen der jeweils Beteiligten voraus. Unter der Perspektive des Athleten sieht Sport nun einmal anders aus als unter der des Zuschauers; für den Wissenschaftler ergeben sich andere Perspektiven als für den Politiker, für die Funktionäre andere als für die, die Sport als Beruf betreiben. Ob es einen gemeinsamen und vor allem auch noch überzeitlichen Nenner für so unterschiedliche Interessen und diese möglicherweise reflektierenden Bedürfnisse gibt, ist sehr fraglich; zumindest ist anzunehmen, daß die Teilbereiche des Sports nur im Feld unterschiedlicher Erwartungen, 
Einleitung in die „Sportwissenschaft ${ }^{\star}$

Interessen, Wünsche, Bedürfnisse der jeweils beteiligten Individuen, Gruppen und Institutionen angemessen bestimmt werden können, so daß sich der Sportwissenschaft $\mathrm{z}$ wingend die Frage stellt, ob die gleichsam unhistorische, auf ein feststehendes Wesen der Leibesübungen rekurrierende Betrachtung des Sports nicht einer an anderen Prämissen orientierten Betrachtungsweise Platz zu machen hat.

\section{Einige Prämissen sporttheoretischer Betrachtungsweisen}

Geht man, wie es die "Sportwissenschaft" zu tun beabsichtigt, davon aus, daß es den „Sport" nicht gibt, so werden damit im Grunde zwei Prämissen unterstellt, die wenigstens genannt werden müssen. Sie beziehen sich darauf,

- daß soziale Bereiche wie Sport und Leibeserziehung nicht ein für allemal zu bestimmen sind, unabhängig von ihrem jeweiligen sozialen, kulturellen Kontext - gleichsam als anthropologisch unveränderliche Kategorie (als solche mag man bestenfalls das Spiel akzeptieren) -, sondern daß sie in ihren Möglichkeiten, Sinndeutungen, Wertsetzungen, Funktionen bestimmt sind oder wenigstens mitbestimmt sind von der jeweiligen soziokulturellen und sozioökonomischen Situation;

- daß Sport und Leibeserziehung historischer Entwicklung unterliegen, keine zeitlosen Werte haben, die sich für alle Zeit gleich bleiben, sondern daß sich Werte, Bedeutungen und Strukturen im historischen Zusammenhang verändern (was die Wichtigkeit der historischen Fragestellung in besonderem Maße unterstreicht).

Wenn diese Prämissen richtig sind, so heißt dies, daß der Sport gegenüber den Grundstrukturen einer Gesellschaft, deren soziale Widersprüche und Konflikte sich auch im Bereich des Sports wiederfinden, eben keinen abgeschirmten oder abschirmbaren Schonraum darstellt, sondern in seinen Produkten, seinen Ergebnissen - wenn man etwa Gesundheit, Leistungsfähigkeit oder auch sportliche Spitzenleistungen und Rekorde als Produkte interpretiert - ähnlichen Verwertungs- und Verwendungszusammenhängen unterliegt wie die von Wissenschaft oder Industrie z. B. auch.

Für die „Sportwissenschaft" hat das eine Reihe von Konsequenzen: Je mehr deutlich wird, daß solche Prämissen im dunkeln liegen, entweder undiskutiert angenommen oder aber ignoriert werden, ist Aufklärung über sie erforderlich; soweit

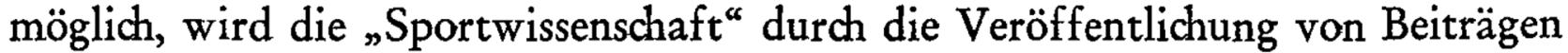
dabei mithelfen; denn nur unter Offenlegung aller Prämissen und Vorentscheidungen, gleichviel welcher Art, die in theoretische und empirische Fragestellungen eingehen, ist es möglich, die unsichere Ebene zufälliger Urteile und Erfahrungen, 


\section{Ommo Grupe}

Vermutungen und Uberinterpretationen in Richtung auf verläßlichere Ergebnisse und Aussagen zu verlassen. Die entscheidendere Konsequenz jedoch ist diese: daß sich Analyse und Reflexion nicht - dies eben deuten die Prämissen an - auf den Sport bzw. seine Teilbereiche beschränken können, sondern immer auch der Hinwendung auf das soziale System bedürfen, innerhalb dessen Sport betrieben, organisiert, verwaltet wird.

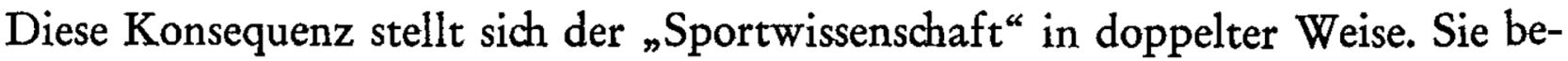
zieht sich nicht allein darauf, wie also die Funktionen des Sports im Rahmen gesellschaftlicher Zusammenhänge und Gruppen zu bestimmen sind, sondern auch darauf, wie Sportwissenschaft selber ihre eigenen sozialen Bezugszusammenhänge bedenken und die Verwendung und Inanspruchnahme der von ihr hervorgebrachten Ergebnisse für welche - eben nicht beliebigen und den Forschungsprozeß irrelevanten - Zwecke mitreflektieren kann. Diese Frage geht darüber hinaus, über das Problem nachzudenken, wie Sportwissenschaft sich in ihren Fragestellungen, Methoden, Verfahren und Leistungen als Wissenschaft zu konstituieren vermag, d. h. diejenigen Bedingungen erfüllt, die es gerechtfertigt erscheinen lassen, sie als Wissenschaft zu bezeichnen, sondern bezieht sich auch auf die Bestimmung ihrer Funktionen im Rahmen gesellschaftlicher Entwicklungen und Reformen, deren Bedingungen und Ziele. Derzeit wird dieses Problem unter der Überschrift „emanzipatorisches" Wissenschaftsverständnis diskutiert, wobei allerdings der Begriff des Emanzipatorischen durch vielfältigen Gebrauch zum Schlagwort degeneriert ist - frei für beliebige Verwendung. Immerhin läßt sich inhaltlich aus dieser Diskussion so viel festhalten, daß Wissenschaften sich die Frage gefallen lassen müssen, was ihre Ergebnisse dem Menchen nützen, wie sie - wenn man so pauschal und banal zugleich fragen will - zu seinem Glück beitragen können.

Solcher Anspruch setzt die Offenheit der wissenschaftlichen Grundauffassung voraus, auch den Verzicht auf Irrationalismen, auf dogmatische Urteile, Parolen und Besserwisserei, auf Disqualifizierung mißliebiger Argumente und Unterdrückung des Widerspruchs. „Sportwissenschaft “ wird sich bemühen, solchen Kurs $\mathrm{zu}$ halten und zu einem Organ nationaler und internationaler Diskussion zu werden, undogmatisch, freimütig, unbefangen, kritik- und konfliktfreudig.

Dabei wird die "Sportwissenschaft" wohl noch geraume Zeit davon ausgehen müssen, daß eine Theorie des Sports ebenso wie eine Theorie der Sportwissenschaft noch aussteht. Weder die Euphorie und Heilserwartung, die sich derzeit mit empirischer Forschung verbindet, noch die durch empirische Skrupel unbefleckten philosophischen Höhenflüge vermögen ihr bei dem Aufbau einer solchen Disziplin

Abb. $1 \Delta$ (Fortsetzung) 
$\mathrm{zu}$ helfen. Sie bedarf vielmehr gediegener empirischer Forschung auf allen Gebieten und einer gescheiten sozial-orientierten Philosophie der individuellen und gesellschaftlichen Implikationen des Sports. Und weiter in einer Phase zunehmender Wissenschaftsgläubigkeit und hochgeschraubter Erwartungen an Wissenschaftler einer erheblichen Skepsis gegenüber beiden.

\section{Interdisziplinäre Gebversuche}

Freilich bleibt solche Diskussion, welches Ergebnis sie auch immer anstrebt, weitgehend formal; über die inhaltliche Seite des Problems einer Sportwissenschaft gibt sie noch keinen Aufschluß. Zwar werden Fragen der wissenschaftstheoretischen Begründung sportwissenschaftlicher Disziplinen - verständlicherweise vor allem der Leibeserziehung - seit Jahren, eigentlich seit Jahrzehnten schon diskutiert, wenngleich auf unterschiedlichem Niveau und mit unterschiedlichen Ergebnissen. Wichtige Zuordnungsfragen blieben indes unbeantwortet. Wohin z. B. gehört die Sportwissenschaft? Wenn sie schon keiner Einzelwissenschaft zuzurechnen ist (was z. B. von der Sportpädagogik mit ihren engen Verflechtungen mit der Erziehungswissenschaft nicht schon ohne weiteres gesagt werden kann, auch nicht von der Sportmedizin), sondern als eine Art Querschnitts- oder Integrationswissenschaft begriffen werden soll (eine gängige Formel, auf die sich ja auch das Herausgeberkollegium, wenn auch mit eingestandener Reserve, eingelassen hat), die sich von dem Gegenstand aus, den sie behandelt, konstituiert - wie dann ist sie zu konzipieren in Methode und Fragestellung und wie kann dies in einer Publikation des Namens „Sportwissenschaft ${ }^{\text {“ }}$ zum Ausdruck gebracht werden?

Mit der Behauptung einer Querschnittswissenschaft wird zunächst nur ein gemeinsamer Gegenstand unterstellt, nicht aber wird eine Gemeinsamkeit der Methoden und der Theoriebildung beansprucht. Aber selbst die Gemeinsamkeit des durch ein gemeinsames Forschungsobjekt umgrenzten Gegenstandsbereichs ist keineswegs schon eindeutig; immerhin handelt es sich beim Sport um einen Bereich, der in sich vielschichtig, in Teilbereiche zerfallen, in der Beurteilung kontrovers ist; selbst auch in ständiger Veränderung begriffen, die wiederum abhängig ist von kaum analysierten, zumeist vermuteten, oft unterstellten Bedingungen sozialer, politischer und ökonomischer Art. (Die Entwicklung des Hochleistungssports $\mathrm{zu}$ einem Teil der Unterhaltungsindustrie, seine zu beobachtende Trennung vom Breitensport, der damit verbundene Trend zu Professionalisierung 


\section{Ommo Grupe}

und Kommerzialisierung, seine bevorzugte Inanspruchnahme für politische Zwecke zeigen beispielhaft die vielfältigen Verflechtungen $z$ wischen einem Teilbereich des Sports und dem jeweiligen sozialen System, das aufgrund seiner besonderen Strukturen, seiner Traditionen und sportpolitischen Planungen in Korrespondenz $\mathrm{zu}$ spitzensportlichen Entwicklungen bestimmte Tendenzen begünstigt, andere abblockt oder unterdrückt.)

Mit dem Anspruch, eine integrative Wissenschaft zu sein, eine Wissenschaft also, die zum Teil unterschiedliche Fragestellungen und (teilweise divergierende) Ergebnisse zusammenfaßt, ist zugleich die Interdisziplinarität der Forschungsansätze vorgegeben, wiederum eine Formel, die sich derzeit innerhalb der Methodendiskussion der Sportwissenschaft nachdrücklich behauptet, wobei Interdisziplinarität voraussetzt, daß die Problemstellungen einer Wisssenschaft von ihrem Objekt aus vorgegeben werden, nicht aber von ihren jeweils spezifischen Methoden und Verfahren, was sie unmittelbar in die einzelwissenschaftlich-isolierte Problemstellung zurückführen würde. Die Betonung des integrativen Moments für den Aufbau der Sportwissenschaft und die Betonung der Notwendigkeit interdisziplinärer Forschungsansätze stehen insofern in engem Zusammenhang.

Freilich - auch Interdisziplinarität ist in der Sportwissenschaft erst noch zu erstreben; bislang sind die ihr angemessenen Methoden, Verfahrensweisen, Fragestellungen noch nicht entwickelt; schließlich ist sie ja auch nicht allein schon auf diese zu begründen, sondern bedarf entsprechender Verhaltensweisen der Forscher selber, die mit Stichworten wie Teamwork und Fähigkeit zu kooperativem Arbeitsstil nur ungenau beschrieben sind. Wer behauptet, die Zeit der Schreibtischforschung sei vorbei, muß Sportwissenschaftler zugleich auf neue forscherische Verhaltensmuster hin beanspruchen, die allerdings (abgesehen von den Medizinern) von den meisten der derzeit Tätigen noch nicht beherrscht werden. Durch die Veröffentlichung von aus interdisziplinärer Zusammenarbeit entstandenen Arbeiten will die „Sportwissenschaft" solche Tendenzen fördern.

Ein erster Schritt dazu ist das Spektrum der Veröffentlichungen, die für die „Sportwissenschaft " vorgesehen sind; es soll neben sportpädagogischen, -psychologischen, -soziologischen, -historischen und -medizinischen Arbeiten im engeren Sinne, Arbeiten zur Biomechanik, Bewegungsforschung (Kinesiologie) und Sensomotorik auch Arbeiten zu sportrelevanten Themen aus den Gebieten der Anthropologie und Philosophie, der Psychiatrie und Verhaltensforschung umfassen; angestrebt wird dabei ein ausgewogenes Verhältnis von theoretischen und empirischen Arbeiten. 
Ein solches relativ breites Spektrum stellt an die Kompetenz derjenigen, die Beiträge auswählen oder vorschlagen, hohe fachliche Anforderungen; dem sollte durch die (noch nicht vollständige) Zusammensetzung des Herausgeberkollegiums und der (ebenfalls nicht vollständigen) Gruppe der korrespondierenden Mitglieder Rechnung getragen werden. Das Herausgeberkollegium hat sehr wohl die Gefahr gesehen, die in einem breiten Ansatz liegt; sie schien ihr jedoch geringer zu sein als die Gefahr, die eine vorzeitige Aufspaltung und Isolierung der sportwissenschaftlichen Teilgebiete mit sich brächte, ehe die Konsolidierung des sportwissenschaftlichen Gesamtbereichs selber zu einem - wenn auch vorläufigen - Abschluß gebracht ist.

\section{Sportwissenschaft oder Sportwissenschaften - Streit um Worte?}

Obber eine angemessene Benennung der geplanten Publikation, die präzise genug, zugleich gebührend skeptisch-zurückhaltend gegenüber zu erwartenden wissenschaftlichen Leistungen, doch auch hinlänglich anspruchsvoll im Blick auf das vorgegebene Gegenstandsfeld und seine interdisziplinäre Behandlungsfähigkeit sei, hat das Herausgeberkollegium lange diskutiert. Zur Debatte standen lediglich die Begriffe Sportwissenschaften und Sportwissenschaft. Noch vor zwei Jahren gab es eine starke Fraktion, die für den ersten Begriff plädierte: Er schien ihr die größere Offenheit gegenüber differierenden Disziplinen zu implizieren, unprätentiös, der Vielfalt der im Sportbereich möglichen wissenschaftsmethodischen Ansätze angemessen, fernab von Ideologieverdacht, auch dem derzeitigen Stand sportwissenschaftlicher Forschung entsprechend, ohne Ansprüche vorzugeben, die durch Leistungen nicht abgedeckt werden könnten, und zudem den Trugschluß abwehrend, es gäbe schon so etwas wie eine einheitliche Sportwissenschaft. Auch hätte der Titel im Plural eher auf die wissenschaftliche Vielfalt der „Sportwissenschaft $^{\text {" }}$ aufmerksam gemacht, der ja nicht nur eine Vielfalt des Gegenstandsbereichs signalisiert, sondern auch die Differenziertheit der ihm angemessenen Fragestellungen und Methoden. Die Argumentation eines der Gutachten (von H. LENK) zur Frage der Benennung liegt auf der Linie dieser Argumente; auch der Geschäftsführende Herausgeber hat sich ursprünglich diesen Argumenten angeschlossen.

Unter dem Einfluß der Entwicklung der letzten Jahre - nämlich der sich verbreitenden (und gewiß zum Teil unreflektierten) Verwendung des Begriffs „Sportwissenschaft" (z. B. Bundesinstitut für Sportwissenschaft, Lehrstühle/Professuren für Sportwissenschaft, Diplome, Promotionen in Sportwissenschaft), seit langem 


\section{Ommo Grupe}

auch in der DDR - hat zwar das Gewicht der Argumente zugunsten der Benennung "Sportwissenschaften“ nicht nachgelassen, wohl aber ist ihr Einfluß geringer geworden, womit die bereits in den ersten Diskussionen vorgetragenen gutachtlichen Äußerungen zugunsten der Singularverwendung (BERNETT, RöHRs) stärker zur Geltung kamen. Bei aller auch von ihnen geteilten Skepsis gegenüber den bisherigen Erträgen sportwissenschaftlicher Forschung heben BERNETT und RöHRs den projektiven Charakter des Begriffs Sportwissenschaft hervor, nämlich die Sicht auf den Sport als einen Forschungsbereich, der unter den Aspekten verschiedener Wissenschaften untersucht wird, wie dies z. B. auch in der Erziehungswissenschaft der Fall ist, die sich mehr und mehr als Sozial- und Verhaltenswissenschaft unter konstitutiver Mitarbeit benachbarter wissenschaftlicher Disziplinen etabliert; die Tendenz, die dahintersteht, ist die Konstituierung von Wissenschaften, ausgehend von dem Gegenstandsbereich, der die wissenschaftlichen Fragestellungen und die Art ihrer Verknüpfung und Kopplung gleichsam nahelegt. Gerade im Sport ist davon auszugehen, daß es keine in sich abgeschlossene Wissenschaft mehr gibt, die mit ihren fachspezifischen Methoden allein, d. h. ohne Kooperation mit anderen wissenschaftlichen Disziplinen, zu seiner Behandlung fähig wäre. Die mehr oder weniger zufällige „Addierung“ (RöHRs) würde zur Unverbindlichkeit führen; erforderlich ist stattdes eine Differenzierung in der Integration (

Freilich - Autonomie, Eigenständigkeit, Einheitlichkeit der Sportwissenschaft (BERNETT) werden mit solcher Argumentation nicht unterstellt, schon gar nicht ist intendiert, unentwickeltes wissenschaftliches Prestige und mangelnde wissenschaftliche Reputation durch Verwendung eines plakativen Begriffs zu kompensieren. Wenngleich die Phase vorwissenschaftlicher Gründerjahre, der Ressentiments wissenschaftlich Unterprivilegierter wie auch versteckter ideologischer Prämissen (BERNETT) überwunden scheint, der Anfang ernst zu nehmender sportwissenschaftlicher Arbeit dagegen durchaus schon sichtbar ist, so sollte im Titel doch weniger der Status quo als ein zu erstrebendes Ziel zum Ausdruck kommen; deshalb das Plädoyer für „Sportwissenschaft“ im Sinne einer „integrierenden Wissenschaft", die die relevanten Ergebnisse anderer wissenschaftlicher Disziplinen verarbeitet, zusammenfaßt und unter ihrer spezifischen Fragestellung zu einem zusammenhängenden - wenngleich wohl nicht oder nie widerspruchsfreien Ganzen integriert, aber auch selber genügend Substanz für wissenschaftliche Eigenleistung aufbringt.

Voller Skepsis gegen ihre eigenen Argumente, doch entschlossen, der Sportfor- 
Einleitung in die „Sportwissenschaft ${ }^{\circ}$

schung auf den Weg zu helfen, hat sich das Herausgeberkollegium also für den Titel "Sportwissenschaft" entschlossen - Programm und noch einzulösender Anspruch zugleich.

\section{Unabbängigkeit der Herausgeber und Autoren - Wissenschaftler als Primadonnen}

Selbstverständlich und wie es sich heute gehört, hat das Herausgeberkollegium auf seine Unabhängigkeit und die der Autoren in Meinung, Auffassung und Kritik bestanden; selbstverständlich ist sie von den nominellen Herausgeberorganisationen - Deutscher Sportbund und Ausschuß Deutscher Leibeserzieher verbindlich und uneingeschränkt zugesichert (was den sonst?). Tatsächlich ist die Absicherung solcher Unabhängigkeit angesichts eines Objekts, das sich finanziell kaum selbst zu tragen vermag, keine sekundäre Frage; und sie ist auch keine Frage, deren Lösung, sollte es einmal zu Konflikten kommen, allein auf gegenseitiges Vertrauen begründet werden könnte, sondern der festen Fundierung in Absprachen bedarf. Auf solcher Basis ist wissenschaftliche Planung möglich, die nicht auf privat-persönliche oder Gruppeninteressen Rücksicht nehmen muß. Im übrigen wird eine solche Publikation, in der der Sport sich in kritischer Verfremdung erkennen kann, ihm nach innen und außen gut anstehen.

Zur Ergänzung des Herausgeberkollegiums konnten eine Reihe ausländischer Kollegen gewonnen werden; sie sollen der "Sportwissenschaft" weniger ein dekoratıves internationales Kolorit verleihen, sondern vor allem dazu beitragen, internationale Kommunikation zu vermitteln und Anschluß an die internationale Forschungsentwidklung zu gewinnen. Mit einer Reihe von Kollegen sind zur Zeit noch Verhandlungen im Gang; es ist zu hoffen, daß ihr Kreis bald auch durch Kollegen aus den osteuropäischen Ländern ergänzt werden kann.

Aus verständlichen Gründen wird „Deutsch" nicht die einzige Sprache der „Sportwissenschaft" bleiben; englische (und gegebenenfalls französische) Manuskripte sollen im Original veröffentlicht werden. Die „Summaries" erscheinen ohnehin dreisprachig.

\section{Themen und Themenplanung}

Zwar werden nicht alle Hefte der "Sportwissenschaft" unter ein Schwerpunktthema gestellt werden, und nicht jedes Schwerpunktthema wird ein Heft vollständig ausfüllen; jedoch sollen nach Möglichkeit bestimmte Themengruppen gebündelt werden. So wird sich z. B. die nächste Nummer der Sportwissenschaft mit dem Schwerpunktthema Sportdidaktik und Curriculumtheorie befassen, das dritte Heft mit Fragen der Bewegungsforschung (Sensomotorik, Kinesiologie) und Trai- 


\section{Ommo Grupe}

ningslehre; anläßlich der Olympischen Spiele in München bietet sich eine ideologiekritische Betrachtung der Olympischen Idee und des Olympismus, des Hochleistungssports und des Amateurproblems an.

Regelmäßig erscheinen Sammelbesprechungen und Literaturberichte über Publikationen innerhalb von Teilgebieten der Sportwissenschaft; vor allem auch ausländische Veröffentlichungen sollen auf diesem Weg bekanntgemacht werden. Außerdem werden regelmäßig Berichte über abgeschlossene und laufende Forschungsprojekte veröffentlicht werden, fortlaufend auch Kongreßankündigungen und Kongreßberichte. Besonderes Gewicht wollen Herausgeberkollegium und Redaktion auf den Rezensionsteil der „Sportwissenschaft“ legen, da sich vor allem in Rezensionen der Reflexionsstand einer wissenschaftlichen Disziplin und das Niveau einer Zeitschrift wiederspiegeln.

Alles in allem wird mit der „Sportwissenschaft" eine Veröffentlichung gestartet, die allen wissenschaftlich arbeitenden und orientierten Kollegen Gelegenheit zur - wenn erforderlich schnellen - Publizierung ihrer Forschungsergebnisse, zu Meinungsaustausch und Kommunikation gibt, den jüngeren zudem die Chance, sich $z \mathfrak{u}$ profilieren und über den engeren Kreis der eigenen Hochschule hinaus bekannt zu werden; dem Sport selbst ein kritisches Organ, für das man ihm die nötige Sensibilität wünschen möchte.

Freilich - ohne kritische Resonanz bei den Lesern wie auch den gegenwärtigen und zukünftigen Mitarbeitern wird das alles nichts werden; sie stellt einen der entscheidenden Faktoren für die weitere Entwicklung der "Sportwissenschaft" dar. Guter Wille und Entschlossenheit der Redaktion und des Herausgeberkollegiums allein reichen nicht aus, ihr auf längere Sicht mit den Seiten auch Substanz zu geben.

Abb. 1 A (Fortsetzung)

Allerdings, so meinen Kritiker nicht unberechtigt, finden sich unter diesen Beiträgen kaum solche, die den Anspruch sportwissenschaftlicher Arbeiten (noch) erreichen, so dass sie Ommo Grupes Verständnis darüber gerecht würden. Zu erörtern wäre, ob diese Beiträge den Anspruch sportwissenschaftlicher Interdisziplinarität schlecht einlösen, ob sie sich ihn erst gar nicht zum Ziel genommen haben, oder ob Sportwissenschaft - die Disziplin genauso wie die zugehörige Zeitschrift - heute vielleicht ganz anders gedacht werden muss?
Dazu ist es beispielsweise wertvoll zu betrachten, welche Personengruppe eigentlich (überwiegend) in der Sportwissenschaft publiziert. Es sind dies mit großer Mehrheit Wissenschaftlerinnen und Wissenschaftler, die sich in Qualifikationsphasen ihrer akademischen Karriere befinden (Doktoranden, Post-Doktoranden), zuweilen unterstützt von (ihren) Professorinnen und Professoren. Viele dieser sich akademisch Qualifizierenden sind in Drittmittelprojekten mit definierten (kürzeren) Vertragslaufzeiten (oft nur für zwei Jahre) ausgestattet. Der Anteil derer, die auf Strukturstellen zumin- dest mittelfristig (z. B. drei-plus-drei Jahre) angestellt sind und die für die Sportwissenschaft schreiben wollen oder könnten, dürfte verhältnismäßig geringer geworden sein. Die Notwendigkeiten des Drittmittelgeschäfts beeinfluss(t)en nicht nur die Personalstrukturen, sondern wirken sich natürlich auch darauf aus wie publiziert wird. Ein großer Teil derer, die sich heutzutage sportwissenschaftlich qualifizieren wollen, sieht sich angesichts der Vertragslaufzeiten und beruflich prekären Umständen möglicherweise gar dazu „gezwungen“ schnell zu publikationsfähigen Ergebnissen bzw. Publikationen 
zu gelangen. Verschärfend kommt hinzu, dass Arbeit in zeitlich eng beschränkten Drittmittelprojekten thematisch und disziplinär oft auch eng begrenzt ist, vielleicht sogar enger begrenzt sein muss. Aus solcherart disziplinär zugespitzter Arbeit entsteht respektable, auf den Sport (im allerweitesten Sinne) bezogene Wissenschaft. Davon zeugen zahlreiche in der Sportwissenschaft aber auch anderswo publizierte Beiträge.

Das von Ommo Grupe für die Sportwissenschaft ins Auge gefasste Ziel, dass in der Zeitschrift ein breites Spektrum an sportwissenschaftlich relevanten Disziplinen aufgefächert mögen werde, könnte also als erreicht markiert werden. Die wünschenswerte Folge daraus, dass so eine Basis von Sportwissenschaft entstehen möge, in der die einzelnen Beiträge mehr als nur das gemeinsame Wort Sport verbinde (welche dann ihrerseits originär sportwissenschaftliche Forschung erst ermögliche) ist demgegenüber ausgeblieben.

Im Rückblick der letzten 44 Jahre betrachtet spricht einiges dafür, dass dem Auffächern der Grundlagen- und Fachdisziplinen eher eine andauernde Phase der zunehmenden Spezialisierung sogar innerhalb der jeweiligen Fachdisziplinen folgte, die eben nicht zu zunehmender Verzahnung und Ineinanderführung anregte. Die Kräfte, die sich in der Sportwissenschaft, sowohl in der wissenschaftlichen Disziplin als auch der Zeitschrift entfalteten und sich auf das Zusammenbinden der fachdisziplinären Betrachtungen richteten, waren offensichtlich weniger stark als die Kräfte, die in Richtung einer Ausdifferenzierung und zunehmenden Verkapselung von Spezialdisziplinen drängten. Sicherlich liegen dieser Entwicklung noch weitere Bedingungsfaktoren, als nur der oben genannte (zunehmende Bedeutung des Drittmittelgeschäfts an Universitäten und daraus resultierende Personalstrukturen) zugrunde. Jedoch mag schon dieser Grund genügend Erklärungskraft bieten, um die Entwicklung der Zeitschrift und ausbleibende Rückwirkungen auf das Fach Sportwissenschaft nachzuvollziehen. Was folgt daraus?

Die gesehene Entwicklung kann man entweder akzeptieren und sich mit ihr arrangieren oder man kann versuchen ihr etwas entgegen zu setzen. Dazu wird die Kraft Einzelner nicht ausreichen, und möglicherweise ist die Vorstellungskraft Einzelner auch nicht genug, vernünftige von den weniger vernünftigen Handlungsalternativen zu unterscheiden.

\section{Ein neuer wissenschaftlicher Beirat der Sportwissenschaft}

Am Ende seines Gründungseditorials formuliert Ommo Grupe: „Freilich - ohne kritische Resonanz bei den Lesern wie auch den gegenwärtigen und zukünftigen Mitarbeitern wird das alles nichts werden; sie stellt einen der entscheidenden Faktoren für die weitere Entwicklung der Sportwissenschaft dar. Guter Wille und Entschlossenheit der Redaktion und des Herausgeberkollegiums allein reichen nicht aus, ihr auf längere Sicht mit den Seiten auch Substanz zu geben“.

Vor allem rufen wir deshalb diejenigen, die sich Willens und besonders gut dazu in der Lage sehen die Sportwissenschaft in ihrem ursprünglich gemeinten Sinne zu bereichern, insbesondere unsere Kollegen Professorinnen und Professoren, dazu auf, sich viel stärker als in den zurückliegenden Jahren für Manuskripte in dieser Zeitschrift zu engagieren; und insbesondere auch eigene einzureichen.

Gemeinsam mit den herausgebenden Institutionen haben wir im Herausgeberkollegium Maßnahmen ergriffen, die der Zeitschrift hoffentlich gleichermaßen zu Gute kommen werden. Nachdem gleich Anfang dieses Jahres der wissenschaftliche Beirat, der die Sportwissenschaft über sehr viele Jahre begleitete, in Ehren verabschiedet wurde, wurde im Februar ein neuer wissenschaftlicher Beirat berufen. Ihm gehören im Forschen und Schreiben erfahrene Kolleginnen und Kollegen aus dem In- und Ausland an. Von diesen erhoffen wir uns unter anderem, dass sie der Sportwissenschaft wirksame Impulse geben können. Nach Berufung zugesagt haben die Professores Herzog, Schwameder, Wank (Biomechanik), Hofmann, Pfister (Sportgeschichte), Baca, Wiemeyer (Sportinformatik), Banzer, Urhausen (Sportmedizin), Beek, Hossner (Sportmotorik), Beech, Franck, Horch (Sportökonomie/-management), Brettschneider, Kurz, O’Sullivan, Prohl (Sportpäd-
agogik/Erziehungswissenschaften), Loland, McNamee, Schürmann (Sportphilosophie), Digel, Fasting, Hartmann-Tews, Maguire (Sportsoziologie), Raab, Williams (Sportpsychologie) sowie Behm, Ferrauti, Hohmann und Müller (Trainingswissenschaft).

Wir bitten darum, uns und diesem Ergebnis Vertrauen zu schenken. Der Auswahlprozess ist mit viel Bedacht und nach intensiven Diskussionen geschehen, und mit Blick auf mögliche Unausgewogenheiten können wir nur entgegnen, dass nicht alle, die wir gerne gewonnen hätten, auch bereit waren mitzuwirken.

Gemeinsam mit den genannten Personen wollen wir nun aber versuchen, die Zukunft der Zeitschrift weiter zu gestalten. Ganz sicher wird dies nicht immer in gerader Fortsetzung der von Ommo Grupe vorgedachten Linie geschehen (können). Die Gegenwart stellt gegenwärtige Anforderungen. Jedoch, und so wollen wir den Bogen vom eingangs festgestellten, traurigen Anlass zu den aktuellen Perspektiven schließen, ist es erstaunlich, wie ähnlich sich doch frühere und heutige Herausforderungen an die Sportwissenschaft in vielen Punkten darstellen. Dieses Wort, Herausforderungen, haben wir dabei sehr bewusst gewählt. Denn es verweist auf den Prozess, der um sie zu bewältigen, stattfinden wird. Diesen Prozess werden wir - ergebnisoffen - begleiten. Dazu erbitten wir Ihre konstruktive Unterstützung.

Mit freundlichen Grüßen

Ralf Brand und Claudia Voelcker-Rehage

\section{Korrespondenzadresse}

Prof. Dr. R. Brand

Exzellenzbereich Kognitionswissenschaft

Department für Sport- und

Gesundheitswissenschaft, Professur für Sportpsychologie Universität Potsdam, Am Neuen Palais 10 14469 Potsdam

ralf.brand@uni-potsdam.de 